CLINICAL STUDY

\title{
Circulating lipocalin 2 is associated with body fat distribution at baseline but is not an independent predictor of insulin resistance: the prospective Cyprus Metabolism Study
}

\author{
Xiaowen Liu ${ }^{1}$, Ole-Petter R Hamnvik ${ }^{2}$, Michael Petrou ${ }^{3}$, Huizhi Gong ${ }^{1}$, John P Chamberland ${ }^{1,5}$, \\ Costas A Christophi ${ }^{3,4}$, Stefanos N Kales ${ }^{4}$, David C Christiani ${ }^{4}$ and Christos S Mantzoros ${ }^{1,2,4,5}$ \\ ${ }^{1}$ Division of Endocrinology, Diabetes, and Metabolism, Department of Medicine, Harvard Medical School, Beth Israel Deaconess Medical Center, 330 \\ Brookline Avenue, Feldberg 875, Boston, Massachusetts 02215, USA, ${ }^{2}$ Division of Endocrinology, Diabetes and Hypertension, Department of Medicine, \\ Brigham and Women's Hospital and Harvard Medical School, Boston, Massachusetts, USA, ${ }^{3}$ Cyprus International Institute for Environmental and Public \\ Health in association with Harvard School of Public Health, Cyprus University of Technology, Limassol, Cyprus, ${ }^{4}$ Department of Environmental Health, \\ Harvard School of Public Health, Boston, Massachusetts, USA and ${ }^{5}$ Section of Endocrinology, Boston VA Healthcare System, Boston, Massachusetts, USA \\ (Correspondence should be addressed to C S Mantzoros at Division of Endocrinology, Diabetes, and Metabolism, Department of Medicine, Harvard Medical \\ School, Beth Israel Deaconess Medical Center; Email: cmantzor@bidmc.harvard.edu)
}

\begin{abstract}
Objective: Lipocalin 2 (LCN2 or NGAL), a protein derived from neutrophils, macrophages, adipocytes, and other cells, has been proposed to be a link between obesity and insulin resistance (IR), but animal and cross-sectional human studies have revealed conflicting results. We studied the association of serum lipocalin 2 with anthropometric, metabolic, and cardiovascular risk markers in young healthy men cross-sectionally and, for the first time, prospectively after 2 years of follow-up, with and without adjustment for potential confounders including serum creatinine.

Design: Two hundred and seventy-two participants were randomly selected from the Cyprus Metabolism Study (1056 men, 18 years), of whom 93 subjects participated in the follow-up study 2 years after baseline assessment. Associations were also explored between total and free leptin levels (to serve as positive controls) and anthropometric metabolic variables.

Results: In the cross-sectional study, lipocalin 2 levels were marginally correlated in the unadjusted model with central fat distribution but not with body weight or total body fat mass. After adjusting for age, smoking, activity, body mass index, fat percentage, waist-to-hip ratio, and serum creatinine, no correlation was found with any cardiovascular risk factor. There was no correlation with the homeostasis model assessment of IR (HOMA-IR) at baseline. In the prospective analyses, baseline levels of lipocalin 2 were not predictive of any variables in unadjusted or adjusted models. As expected, total and free leptin were associated with anthropometric and metabolic variables both cross-sectionally and prospectively.

Conclusions: We demonstrate that lipocalin 2 is not an independent predictor of metabolic and cardiovascular risk factors in young men cross-sectionally or prospectively.
\end{abstract}

European Journal of Endocrinology 165 805-812

\section{Introduction}

Lipocalin 2 (LCN2), or neutrophil gelatinase-associated lipocalin (NGAL), is a $25 \mathrm{kDa}$ secretory glycoprotein originally identified in human neutrophils (1). Lipocalin 2 belongs to the larger superfamily of proteins called lipocalins, which also includes retinol-binding protein 4. These small proteins are characterized by a hydrophobic ligand-binding pocket that allows them to function as transport proteins for hydrophobic molecules such as iron, retinoids, and steroids (2). Lipocalin 2 is secreted by adipocytes and immune cells such as neutrophils and macrophages and was initially described as playing a role in innate immunity, perhaps by sequestrating iron (3). In addition, proinflammatory molecules such as lipopolysaccharide and interleukin $1 \beta$ stimulate its expression and secretion $(4,5)$, and lipocalin 2 levels correlate with markers of inflammation such as high sensitivity C-reactive protein (6). This suggests that lipocalin 2 plays a role in immune function and inflammation. As chronic inflammation is a risk factor for the development of metabolic abnormalities that accompany obesity, lipocalin 2 has emerged as a potential link between obesity, inflammation, cardiovascular disease, and obesity-associated metabolic dysfunction such as insulin resistance (IR) (7). Previously published animal studies (7-9) and crosssectional human studies have revealed conflicting 
findings, with one reporting an association between lipocalin 2 and body mass index (BMI), waist circumference and fat percentage, as well as adverse lipid profiles and IR (6), and others failing to find any correlation with BMI (10) or with insulin sensitivity (11). These studies did not correct for renal function. However, renal function is known to affect lipocalin 2 levels, and lipocalin 2 has in fact been studied as a marker of acute kidney injury $(12,13)$. Also, previous studies have only included middle-aged subjects, and to date, there have been no published prospective studies. Thus, the role of lipocalin 2 as a potential metabolic and cardiovascular risk factor remains to be fully elucidated. We studied the association of lipocalin 2 with anthropometric and metabolic markers in young healthy men, both cross-sectionally and prospectively, with and without adjustment for potential confounders including serum creatinine as a marker of renal function.

\section{Subjects and methods}

The full design of the Cyprus Metabolism Study has been published elsewhere (14). We investigated 272 participants from this study. The participants were 18-year-old men, of whom we investigated 93 participants who attended the follow-up assessment 2 years after the initial enrollment and have baseline samples available. In addition, we randomly selected another 179 participants, who participated only in the crosssectional study, by randomly picking samples from the sample containers. Baseline and follow-up anthropometrics and metabolic parameters were measured as described previously (14). In brief, anthropometric measurements and vital signs were obtained twice in a standardized manner by trained technicians, with a third measurement if there was discordance. Body composition was measured using the TanitaTBF-300A Body Composition Analyzer; basal metabolic rate was calculated by the analyzer using a Tanita proprietary formula. BMI was calculated as the weight (in $\mathrm{kg}$ ) divided by the square of the height (in meters). Routine baseline and follow-up biochemical measurements were performed using automated laboratory methods (Olympus AU2700 Chemistry-Immuno Analyzer, Olympus, Center Valley, PA, USA). Baseline serum lipocalin 2 levels were measured in duplicate using ELISA (BioVendor, Candler, NC, USA), with a sensitivity of $0.02 \mathrm{ng} / \mathrm{ml}$, an intra-assay coefficient of variation (CV) of $7.03-8.38 \%$, and an inter-assay CV of 9.73$9.77 \%$. Serum total and free leptin were measured as described previously (15).

Dyslipidemia was defined as any one of the following: total cholesterol above $200 \mathrm{mg} / \mathrm{dl}$, high-density lipoprotein (HDL) cholesterol below $40 \mathrm{mg} / \mathrm{dl}$, low-density lipoprotein (LDL) cholesterol above $130 \mathrm{mg} / \mathrm{dl}$, or triglycerides above $150 \mathrm{mg} / \mathrm{dl}$. Homeostasis model assessment of IR (HOMA-IR) is defined as fasting glucose $(\mathrm{mg} / \mathrm{dl})$ multiplied by fasting insulin $(\mathrm{mU} / \mathrm{l})$, divided by 405 (16). Activity level was assessed using questionnaires and includes both exercise and habitual activities such as walking or farm work.

All the variables were tested for normal distribution and logarithmically transformed if not normally distributed.

Means and s.E.M., or for categorical variables, number and percentage, were determined for the descriptive statistics. Pearson correlation and partial correlation coefficients were obtained between variables in the cross-sectional study. General linear models were used in the prospective study. In the adjusted models, we correct for BMI as a measure of weight/height correlation, body fat percentage as a measure of the amount of the body weight that is fat, and waist-to-hip ratio (WHR) as a marker of body fat distribution. $P<0.017$ was considered statistically significant based on Bonferroni correction for testing three hypotheses (leptin, free leptin, and lipocalin 2) at the same time using one set of data. SAS (version 9.1, SAS Institute, Cary, NC, USA) was used for the statistical analysis. This research was approved by Harvard School of Public Health and the Cyprus National Bioethics Committee.

\section{Results}

\section{Study participants}

Descriptive characteristics of the study population are presented in Table 1. Of note, though the participants on average had normal anthropometrics and cardiovascular risk factors, a large proportion was overweight with a BMI $>25 \mathrm{~kg} / \mathrm{m}^{2}(26.1 \%)$ and $32.0 \%$ had dyslipidemia (mainly elevated LDL or reduced HDLcholesterol).

Table 1 also shows the baseline and follow-up characteristics of the 93 participants who took part in the prospective study. The follow-up group had lower BMI and WHR compared with the 272 subjects who were included in this study at baseline. Most of the anthropometric variables and cardiovascular risk factors significantly worsened over the 2-year period between visits.

\section{Cross-sectional study}

Findings from the cross-sectional study are summarized in Table 2. Serum levels of total and free leptin were significantly strongly associated with anthropometric, cardiovascular, and metabolic variables (all $P<0.01$, most $P<0.0001)$ except for height, serum urea, and creatinine levels in the unadjusted correlation analysis. However, serum lipocalin 2 levels were only marginally correlated with waist circumference $(r=0.15$, 
Table 1 Baseline characteristics of participants in the cross-sectional study and the prospective study. Results are presented as mean \pm s.D. (median (Q1-Q3)) or as $n(\%)$.

\begin{tabular}{|c|c|c|c|c|c|}
\hline \multirow[b]{2}{*}{ Variables } & \multicolumn{3}{|c|}{ At baseline } & \multirow{2}{*}{$\begin{array}{l}\text { At follow-up } \\
\text { Follow-up group } \\
\qquad(n=93)\end{array}$} & \multirow[b]{2}{*}{$\boldsymbol{P}^{\dagger}$} \\
\hline & $\begin{array}{l}\text { All subjects } \\
(n=272)\end{array}$ & $\begin{array}{l}\text { Follow-up group } \\
\qquad(n=93)\end{array}$ & $P^{*}$ & & \\
\hline Age (years) & $18.2 \pm 0.14$ & $18.2 \pm 0.12$ & 0.37 & & \\
\hline Activity (h/week) & $10.9 \pm 12.5$ & $11.3 \pm 13.4$ & 0.66 & & \\
\hline \multicolumn{6}{|l|}{ Anthropometric variables } \\
\hline Height $(\mathrm{cm})$ & $175.0 \pm 6.2$ & $175.0 \pm 6.1$ & 0.59 & $175.2 \pm 6.2$ & $<0.0001$ \\
\hline Weight (kg) & $71.2 \pm 13.7$ & $68.9 \pm 12.8$ & 0.05 & $72.9 \pm 12.9$ & $<0.0001$ \\
\hline $\operatorname{BMI}\left(\mathrm{kg} / \mathrm{m}^{2}\right)$ & $23.2 \pm 3.9$ & $22.5 \pm 3.5$ & 0.04 & $23.7 \pm 3.6$ & $<0.0001$ \\
\hline Total body fat (\%) & $13.8 \pm 6.0$ & $12.8 \pm 5.7$ & 0.05 & $15.6 \pm 6.0$ & $<0.0001$ \\
\hline Fat mass $(\mathrm{kg})$ & $10.6 \pm 6.7$ & $9.5 \pm 6.4$ & 0.05 & $12.1 \pm 7.5$ & $<0.0001$ \\
\hline Fat-free mass (kg) & $60.6 \pm 7.7$ & $59.4 \pm 7.1$ & 0.07 & $60.9 \pm 6.5$ & $<0.0001$ \\
\hline Waist circumference $(\mathrm{cm})$ & $81.2 \pm 10.5$ & $79.1 \pm 9.4$ & 0.02 & $83.8 \pm 9.7$ & $<0.0001$ \\
\hline Hip circumference $(\mathrm{cm})$ & $97.0 \pm 8.4$ & $95.7 \pm 7.8$ & 0.06 & $97.1 \pm 7.9$ & 0.0005 \\
\hline Waist-to-hip ratio & $0.83 \pm 0.05$ & $0.82 \pm 0.04$ & 0.01 & $0.86 \pm 0.04$ & $<0.0001$ \\
\hline BMR (kcal/day) & $1802 \pm 206$ & $1770 \pm 195$ & 0.06 & $1814 \pm 197$ & $<0.0001$ \\
\hline \multicolumn{6}{|l|}{ Cardiovascular risk factors } \\
\hline Heart rate (beats/min) & $66.3 \pm 9.2$ & $66.1 \pm 8.9$ & 0.72 & $71.6 \pm 9.6$ & $<0.0001$ \\
\hline SBP $(\mathrm{mmHg})$ & $106.9 \pm 10.6$ & $106.2 \pm 10.8$ & 0.48 & $111.7 \pm 9.6$ & $<0.0001$ \\
\hline $\mathrm{DBP}(\mathrm{mmHg})$ & $61.8 \pm 7.5$ & $61.8 \pm 7.3$ & 0.98 & $71.6 \pm 7.2$ & $<0.0001$ \\
\hline Total cholesterol (mg/dl) & $148.5 \pm 28.8$ & $144.3 \pm 25.7$ & 0.08 & $159.6 \pm 27.0$ & $<0.0001$ \\
\hline HDL-cholesterol (mg/dl) & $47.3 \pm 8.9$ & $47.1 \pm 8.4$ & 0.82 & $46.0 \pm 8.5$ & 0.10 \\
\hline LDL-cholesterol (mg/dl) & $105.9 \pm 25.9$ & $101.9 \pm 22.9$ & 0.06 & $98 . .4 \pm 23.2$ & 0.05 \\
\hline Triglycerides (mg/dl) & $61.7 \pm 23.3$ & $58.7 \pm 22.0$ & 0.13 & $75.8 \pm 41.0$ & $<0.0001$ \\
\hline \multicolumn{6}{|c|}{ Hormonal and metabolic variables } \\
\hline Fasting glucose $(\mathrm{mg} / \mathrm{dl})$ & $79.1 \pm 7.0$ & $78.2 \pm 6.7$ & 0.11 & $86.9 \pm 9.4$ & $<0.0001$ \\
\hline Insulin $(\mu \mathrm{U} / \mathrm{ml})$ & $7.8 \pm 3.7$ & $7.4 \pm 3.3$ & 0.22 & & \\
\hline HOMA-IR & $1.53 \pm 0.78$ & $1.44 \pm 0.69$ & 0.16 & & \\
\hline Leptin (ng/ml) & $\begin{array}{c}2.14 \pm 2.24 \\
(1.27(0.76-2.53))\end{array}$ & $\begin{array}{c}1.76 \pm 1.91 \\
(0.97(0.73-1.77))\end{array}$ & 0.04 & & \\
\hline Free leptin (ng/ml) & $\begin{array}{c}1.63 \pm 2.16 \\
(0.78(0.45-1.77))\end{array}$ & $\begin{array}{c}1.21 \pm 1.61 \\
(0.60(0.42-1.06))\end{array}$ & 0.02 & & \\
\hline LBP (ng/ml) & $24.5 \pm 5.6$ & $25.0 \pm 4.9$ & 0.30 & & \\
\hline Adiponectin $(\mu \mathrm{g} / \mathrm{ml})$ & $7.1 \pm 2.5$ & $6.6 \pm 1.6$ & 0.08 & & \\
\hline Lipocalin 2 (ng/ml) & $39.1 \pm 16.2$ & $39.9 \pm 13.8$ & 0.54 & & \\
\hline Uric acid (ng/dl) & $6.7 \pm 1.1$ & $6.6 \pm 1.1$ & 0.43 & $6.0 \pm 1.1$ & $<0.0001$ \\
\hline Urea (ng/dl) & $35.8 \pm 6.7$ & $35.8 \pm 6.7$ & 0.90 & $30.4 \pm 6.1$ & $<0.0001$ \\
\hline Creatinine (ng/dl) & $1.1 \pm 0.1$ & $1.05 \pm 0.11$ & 0.76 & $1.1 \pm 0.1$ & 0.002 \\
\hline \multicolumn{6}{|l|}{ Categorical variables } \\
\hline Smoking status & & & 0.03 & & \\
\hline Never & $149(54.8)$ & $60(64.5)$ & & & \\
\hline Previous & $10(3.7)$ & $2(2.2)$ & & & \\
\hline Current & $113(41.5)$ & 31 (33.3) & & & \\
\hline $\mathrm{BMI}>25 \mathrm{~kg} / \mathrm{m}^{2}$ & $71(26.1)$ & $17(18.3)$ & 0.04 & $22(23.7)$ & 0.35 \\
\hline Waist circumference $>94 \mathrm{~cm}$ & 35 (12.9) & $7(7.5)$ & 0.06 & 12 (12.9) & 0.21 \\
\hline Total cholesterol > $200 \mathrm{mg} / \mathrm{dl}$ & $12(4.4)$ & $2(2.2)$ & 0.21 & $7(7.5)$ & 0.09 \\
\hline $\mathrm{HDL}<40 \mathrm{mg} / \mathrm{dl}$ & $53(19.5)$ & $14(15.1)$ & 0.19 & $22(23.7)$ & 0.14 \\
\hline $\mathrm{LDL}>130 \mathrm{mg} / \mathrm{dl}$ & $42(15.4)$ & $12(12.9)$ & 0.41 & $9(9.7)^{\prime}$ & 0.49 \\
\hline Triglycerides $>150 \mathrm{mg} / \mathrm{dl}$ & $1(0.37)$ & $1(1.1)$ & 0.98 & $6(6.5)$ & 0.05 \\
\hline Dyslipidemia & $87(32.0)$ & $26(28.0)$ & 0.31 & $34(36.6)$ & 0.21 \\
\hline
\end{tabular}

${ }^{\star} P$ for difference between entire cohort versus follow-up group at baseline. T-test is used for continuous variables and $\chi^{2}$ tests were used for categorical variables. ${ }^{\dagger} P$ for difference of follow-up group between baseline and 2-year follow-up, repeated measure analyses were applied for both continuous and categorical variables. $P$-values in bold were considered statistically significant after Bonferroni correction $(P<0.017)$. BMI, body mass index; BMR, basal metabolic rate; DBP, diastolic blood pressure; HDL, high-density lipoprotein; HOMA-IR, homeostasis model of assessment-insulin resistance; LBP, leptinbinding protein; LDL, low-density lipoprotein; SBP, systolic blood pressure.

$P=0.017)$, triglycerides $(r=0.18, P=0.004)$, and uric acid $(r=0.15, P=0.008)$, but not with weight, BMI, total body fat mass, WHR, HOMA-IR, or other anthropometric, cardiovascular, and metabolic variables in unadjusted analysis.

After controlling for age, BMI, total body fat percentage, WHR, smoking status, and activity, serum levels of total and free leptin were still strongly associated with fasting glucose $(r=0.22$, $P=0.0003$, and $r=0.21, P=0.0007$ respectively), insulin $(r=0.22, \quad P=0.0003$, and $r=0.24$, $P<0.0001$ respectively $)$, and HOMA-IR $(r=0.25$, $P<0.0001$, and $r=0.27, P<0.0001$ respectively). Serum levels of lipocalin 2 became significantly 


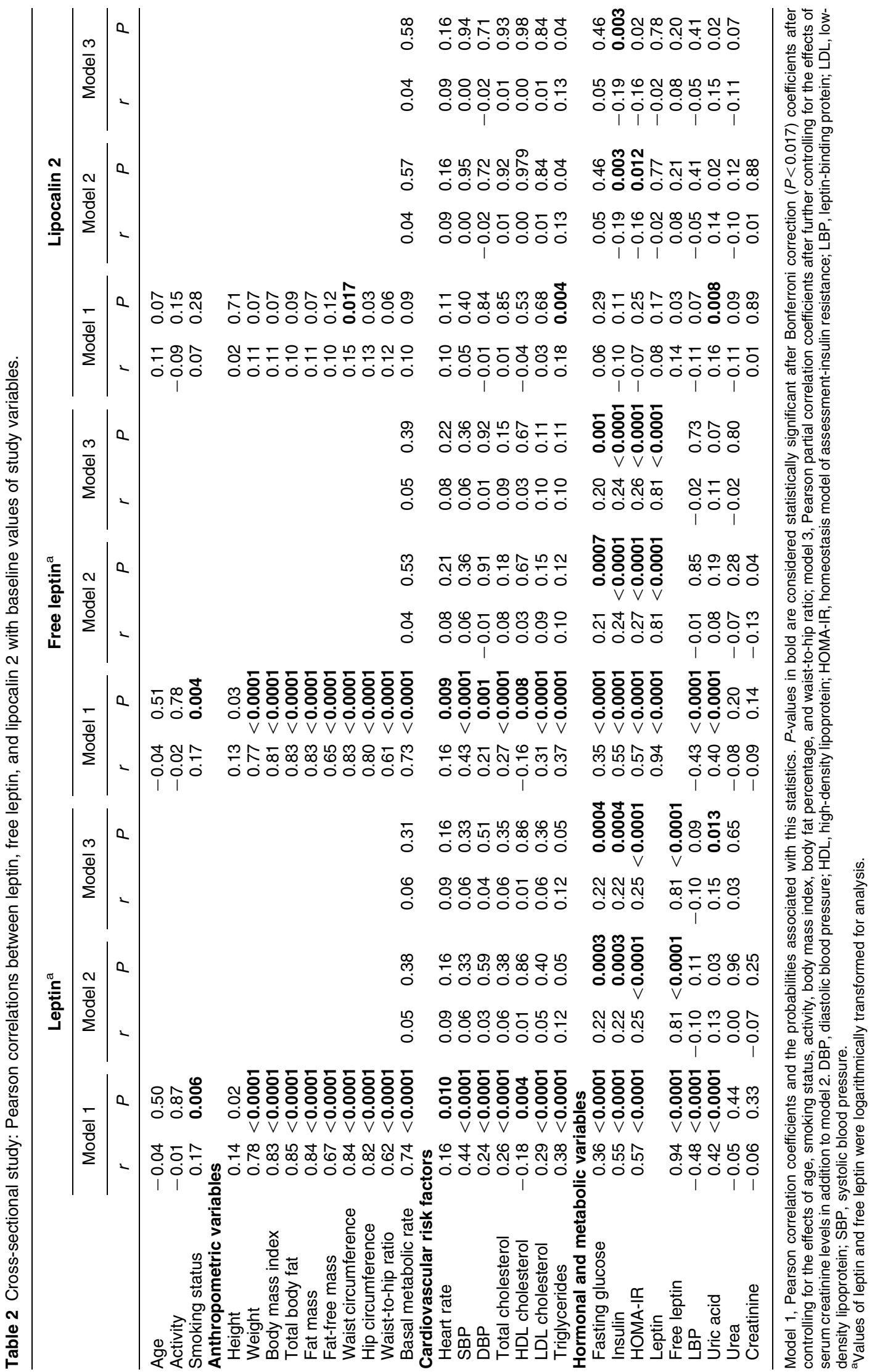




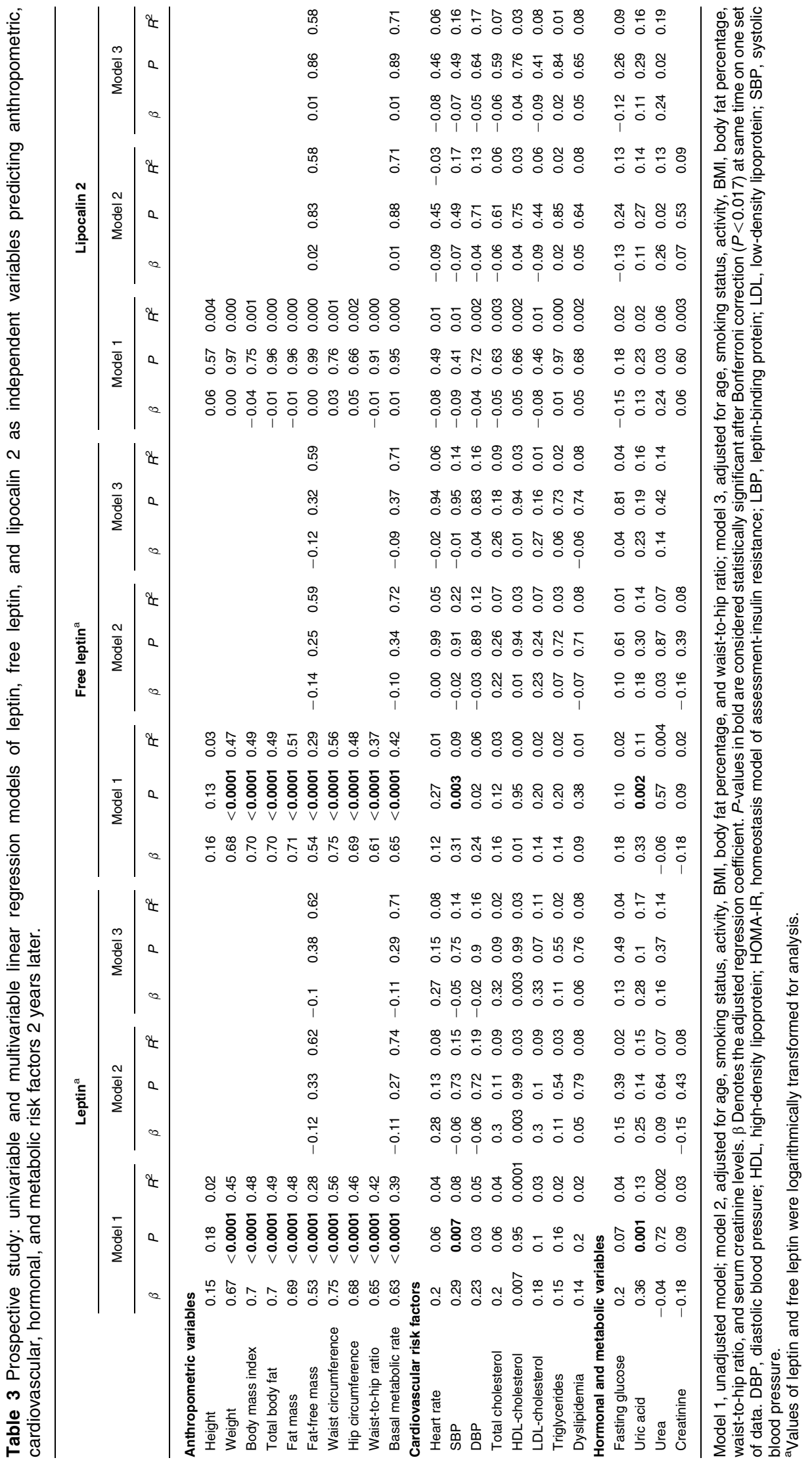


inversely correlated with insulin $(r=-0.19, P=0.003)$ and HOMA-IR $(r=-0.16, P=0.012)$.

After further controlling for serum creatinine levels, the correlation between serum total, free leptin, and the fasting glucose, insulin and HOMA-IR remained significant $(r>0.20, P<0.001$ for all variables). Conversely, the correlation between serum lipocalin 2 and HOMA-IR was no longer significant after controlling for creatinine.

\section{Prospective study}

The results for the prospective study are presented in Table 3. Baseline levels of lipocalin 2 were not predictive at 2-year follow-up for any variables in the unadjusted model; the model adjusted for age, BMI, total body fat percentage, WHR, smoking status, and activity; or the model further adjusted for serum creatinine levels.

Baseline levels of both total and free leptin were significantly predictive of anthropometric variables (all $P<0.0001)$, systolic blood pressure $(P=0.007$ and $P=0.003$ respectively), and uric acid ( $P=0.001$ and $P=0.002$ respectively) in the unadjusted model. After adjusting for age, BMI, total body fat percentage, WHR, smoking status, and activity, and further adjusting for serum creatinine levels, baseline levels of leptin and free leptin were not predictive for any variables at 2-year follow-up.

\section{Discussion}

We demonstrate that lipocalin 2 is weakly associated with waist circumference as a marker of central fat distribution, but not with WHR or total body fat. The correlation with IR disappeared after adjusting for serum creatinine. We also show for the first time that, prospectively, lipocalin 2 is not an independent predictor of metabolic and cardiovascular risk factors in young men.

Mouse studies have suggested contradictory roles for lipocalin 2 in glucose metabolism. Whereas one study found that lipocalin 2 knockout mice had improved insulin sensitivity (8), other studies found that either the global ablation of lipocalin 2 has a minor role in high-fat diet-induced glucose intolerance (17) or that lipocalin gene knockout mice were more susceptible to diet-induced obesity and IR (9). Similarly, previous cross-sectional human studies show contradictory findings. In a study of 33- to 72-year-old men and women, a positive correlation between lipocalin 2 and adiposity, triglycerides, glucose, and HOMA-IR was found (6). These correlations were significant even after adjusting for age, sex, and BMI. However, a study of 58-year-old healthy Swedish men found no correlation between lipocalin 2 and insulin sensitivity, waist circumference, or BMI, although it showed an inverse correlation with HDL- and LDL-cholesterol (11). In comparison, we found that lipocalin 2 was associated more strongly with waist circumference, an imperfect marker of visceral adiposity, than with BMI, a marker of general adiposity. This raises the possibility that different body fat compartments secrete different amounts of lipocalin 2. However, to date, no study has investigated whether lipocalin 2 is differentially expressed in visceral fat versus subcutaneous fat. One study using visceral fat from obese subjects did confirm lipocalin 2 mRNA expression in this tissue, but subcutaneous fat tissue was not studied (10). Interventional studies looking at the effect of weight loss, which is known to improve insulin sensitivity, have mostly found no change in lipocalin 2 levels with weight loss $(18,19)$, although a recent study did find a reduction in women with polycystic ovary syndrome (PCOS) (20). Despite the significant correlation between lipocalin 2 and HOMA-IR at baseline when adjusting for anthropometric measurements, this correlation disappears after also adjusting for serum creatinine. Renal function is known to affect lipocalin 2 levels $(12,13)$. This suggests that the weak correlations between lipocalin 2 and IR seen in previous studies could be related to differences in renal function, in addition to differences in the studied populations in terms of sample size, age, gender, and ethnicity. Previous studies were relatively small and conducted in middle-aged individuals with pre-existing metabolic derangements. Also no previous study has previously corrected for renal function. We do report a weak negative correlation between lipocalin 2 and insulin in the cross-sectional analysis after adjustment, indicating a potential negative effect of lipocalin 2 on $\beta$-cell function. This remains to be studied by future basic research studies. In aggregate, these findings do not support a major etiologic role for lipocalin 2 in IR or obesity.

Our data are consistent with weak/null correlations with insulin sensitivity in previous cross-sectional studies and extend them by demonstrating for the first time null associations prospectively. Strengths of this study include that it is the largest cross-sectional study published to date and the first prospective study examining the associations between serum lipocalin 2 levels and future metabolic characteristics in men. The study also includes the simultaneous assessment of positive controls, leptin, and free leptin. These have been found earlier to correlate closely with cross-sectional and prospective assessments of cardiometabolic risk factors $(21,22)$. The demonstration of expected associations with leptin and free leptin in our study confers internal validity. We also adjusted for known potential confounders. Measurements were performed on de-identified samples by technicians who were blinded to the study hypotheses. Random assay variability could result in misclassification; this would have suppressed effect estimates and therefore would not be responsible for demonstration of significance where such does not exist. 
The limitations of our study include a young, healthy population, and a relatively short follow-up time with a relatively small follow-up group. Renal function was assessed using serum levels of creatinine instead of a direct measurement of glomerular filtration rate or 24-h urinary excretion of creatinine. Although the data are representative of the male population of Cyprus, these data may not be generalizable to other populations.

Larger prospective studies are needed to confirm our data in women and/or older subjects. Moreover, this is an observational study, and interventional, mechanistic studies are needed to confirm or refute our findings that lipocalin 2 may not play a major role in the metabolic syndrome, diabetes, and cardiovascular disease.

\section{Declaration of interest}

The authors declare that there is no conflict of interest that could be perceived as prejudicing the impartiality of the research reported.

\section{Funding}

The study was supported by the Cyprus Research Promotion Foundation (EPYE $\Xi / 0205 / 10$ ). The Mantzoros Lab is supported by a discretionary grant from Beth Israel Deaconess Medical Center.

\section{Author contribution statement}

C S Mantzoros derived the hypothesis and conceived the study design; M Petrou, C A Christophi, S N Kales, and D C Christiani planned and organized the collection of the data; X Liu, H Gong, and J P Chamberland performed the laboratory analyses; X Liu and C S Mantzoros collated the data and planned and performed the statistical analyses; X Liu, O-P R Hamnvik, M Petrou, C A Christophi, S N Kales, D C Christiani, and C S Mantzoros contributed to the interpretation and discussion of results; and X Liu and O-P R Hamnvik wrote the manuscript. This report was critically reviewed and subsequently approved by all authors.

\section{Acknowledgements}

An abstract of this study was presented at the Endocrine Society annual meeting (Endo 2011) in June 2011. We would like to thank the Harvard Cyprus Program, Nicosia General Hospital, and all volunteers for participating in the study, and especially Maria Michael, Androula Chasikou-Constantinidou, and Christos Tapakoudes for their expert technical assistance.

\section{References}

1 Kjeldsen L, Johnsen AH, Sengelov H \& Borregaard N. Isolation and primary structure of NGAL, a novel protein associated with human neutrophil gelatinase. Journal of Biological Chemistry 1993 268 10425-10432.

2 Flower DR. The lipocalin protein family: structure and function. Biochemical Journal 1996318 1-14.

3 Flo TH, Smith KD, Sato S, Rodriguez DJ, Holmes MA, Strong RK, Akira $S$ \& Aderem A. Lipocalin 2 mediates an innate immune response to bacterial infection by sequestrating iron. Nature 2004 432 917-921. (doi:10.1038/nature03104)
4 Liu Q \& Nilsen-Hamilton M. Identification of a new acute phase protein. Journal of Biological Chemistry $199527022565-22570$. (doi:10.1074/jbc.270.38.22565)

5 Jayaraman A, Roberts KA, Yoon J, Yarmush DM, Duan X, Lee K \& Yarmush ML. Identification of neutrophil gelatinase-associated lipocalin (NGAL) as a discriminatory marker of the hepatocytesecreted protein response to IL-1beta: a proteomic analysis. Biotechnology and Bioengineering 200591 502-515. (doi:10. 1002/bit.20535)

6 Wang Y, Lam KS, Kraegen EW, Sweeney G, Zhang J, Tso AW, Chow WS, Wat NM, Xu JY, Hoo RL \& Xu A. Lipocalin-2 is an inflammatory marker closely associated with obesity, insulin resistance, and hyperglycemia in humans. Clinical Chemistry 200753 34-41. (doi:10.1373/clinchem.2006.075614)

7 Yan QW, Yang Q, Mody N, Graham TE, Hsu CH, Xu Z, Houstis NE, Kahn BB \& Rosen ED. The adipokine lipocalin 2 is regulated by obesity and promotes insulin resistance. Diabetes $2007 \mathbf{5 6}$ 2533-2540. (doi:10.2337/db07-0007)

8 Law IK, Xu A, Lam KS, Berger T, Mak TW, Vanhoutte PM, Liu JT, Sweeney G, Zhou M, Yang B \& Wang Y. Lipocalin-2 deficiency attenuates insulin resistance associated with aging and obesity. Diabetes 201059 872-882. (doi:10.2337/db091541)

9 Guo H, Jin D, Zhang Y, Wright W, Bazuine M, Brockman DA, Bernlohr DA \& Chen X. Lipocalin-2 deficiency impairs thermogenesis and potentiates diet-induced insulin resistance in mice. Diabetes 201059 1376-1385. (doi:10.2337/db09-1735)

10 Catalan V, Gomez-Ambrosi J, Rodriguez A, Ramirez B, Silva C, Rotellar F, Gil MJ, Cienfuegos JA, Salvador J \& Fruhbeck G. Increased adipose tissue expression of lipocalin-2 in obesity is related to inflammation and matrix metalloproteinase-2 and metalloproteinase-9 activities in humans. Journal of Molecular Medicine 200987 803-813. (doi:10.1007/s00109-009-0486-8)

11 Wallenius V, Elias E, Bergstrom GM, Zetterberg H \& Behre CJ. The lipocalins retinol-binding protein-4, lipocalin-2 and lipocalin-type prostaglandin D2-synthase correlate with markers of inflammatory activity, alcohol intake and blood lipids, but not with insulin sensitivity in metabolically healthy 58 -year-old Swedish men. Experimental and Clinical Endocrinology \& Diabetes 2011119 75-80. (doi:10.1055/s-0030-1265212)

12 Mishra J, Dent C, Tarabishi R, Mitsnefes MM, Ma Q, Kelly C, Ruff SM, Zahedi K, Shao M, Bean J, Mori K, Barasch J \& Devarajan P. Neutrophil gelatinase-associated lipocalin (NGAL) as a biomarker for acute renal injury after cardiac surgery. Lancet 2005365 1231-1238. (doi:10.1016/S0140-6736(05) 74811-X)

13 Mori K \& Nakao K. Neutrophil gelatinase-associated lipocalin as the real-time indicator of active kidney damage. Kidney International 2007 71 967-970. (doi:10.1038/sj.ki.5002165)

14 Hamnvik OP, Liu X, Petrou M, Gong H, Chamberland JP, Kim EH, Christophi CA, Kales SN, Christiani DC \& Mantzoros CS. Soluble leptin receptor and leptin are associated with baseline adiposity and metabolic risk factors, and predict adiposity, metabolic syndrome, and glucose levels at 2-year follow-up: the Cyprus Metabolism Prospective Cohort Study. Metabolism 201160 987-993. (doi:10.1016/j.metabol.2010.09.009)

15 Chou SH, Chamberland JP, Liu X, Matarese G, Gao C, Stefanakis R, Brinkoetter MT, Gong H, Arampatzi K \& Mantzoros CS. Leptin is an effective treatment for hypothalamic amenorrhea. PNAS 2011 108 6585-6590. (doi:10.1073/pnas.1015674108)

16 Matthews DR, Hosker JP, Rudenski AS, Naylor BA, Treacher DF \& Turner RC. Homeostasis model assessment: insulin resistance and beta-cell function from fasting plasma glucose and insulin concentrations in man. Diabetologia 198528 412-419. (doi:10. 1007/BF00280883)

17 Jun LS, Siddall CP \& Rosen ED. A minor role for lipocalin 2 in high fat diet-induced glucose intolerance. American Journal of Physiology. Endocrinology and Metabolism, 2011 In press. (doi:10. 1152/ajpendo.00147.2011)

18 Corripio R, Gonzalez-Clemente JM, Perez-Sanchez J, Naf S, Gallart L, Nosas R, Vendrell J \& Caixas A. Weight loss in 
prepubertal obese children is associated with a decrease in adipocyte fatty-acid-binding protein without changes in lipocalin-2: a 2-year longitudinal study. European Journal of Endocrinology 2010163 887-893. (doi:10.1530/EJE-10-0408)

19 Choi KM, Kim TN, Yoo HJ, Lee KW, Cho GJ, Hwang TG, Baik SH, Choi DS \& Kim SM. Effect of exercise training on A-FABP, lipocalin2 and RBP4 levels in obese women. Clinical Endocrinology 200970 569-574. (doi:10.1111/j.1365-2265.2008.03374.x)

20 Koiou E, Tziomalos K, Katsikis I, Kandaraki EA, Kalaitzakis E, Delkos D, Vosnakis C \& Panidis D. Weight loss significantly reduces serum lipocalin-2 levels in overweight and obese women with polycystic ovary syndrome. Gynecological Endocrinology, 2011. In press. (doi:10.3109/09513590.2011.588745)

21 Ingelsson E, Larson MG, Yin X, Wang TJ, Meigs JB, Lipinska I, Benjamin EJ, Keaney JF Jr \& Vasan RS. Circulating ghrelin, leptin, and soluble leptin receptor concentrations and cardiometabolic risk factors in a community-based sample. Journal of Clinical Endocrinology and Metabolism 200893 3149-3157. (doi:10. 1210/jc.2008-0207)

22 Franks PW, Brage S, Luan J, Ekelund U, Rahman M, Farooqi IS, Halsall I, O'Rahilly S \& Wareham NJ. Leptin predicts a worsening of the features of the metabolic syndrome independently of obesity. Obesity Research 200513 1476-1484. (doi:10.1038/ oby.2005.178)

Received 6 April 2011

Revised version received 18 August 2011

Accepted 1 September 2011 